\title{
XIX meeting of the Italian Society of Pure and Applied Biophysics
}

\author{
Daniela Giacomazza
}

Received: 24 February 2009 / Accepted: 28 February 2009 / Published online: 3 April 2009

(C) European Biophysical Societies' Association 2009

This special issue of the European Biophysics Journal contains papers presented as invited lectures or oral contributions at the XIX meeting of the Italian Society of Pure and Applied Biophysics, also known as SIBPA. The conference was held in Rome, 17-20 September 2008 at the "Sacro Cuore" Catholic University.

For the second time, the first was in 2006, the articles presented at the conference appear in this journal that is the major point of reference for the European Biophysical Societies' Association (EBSA) and once again witness of the interesting development of the Italian Biophysics.

The main topics of the meeting were organized in seven sessions, with two invited lectures per session, and a special lecture concerning the renewable energies obtained from biological sources performed by J. Barber of the Wolfson Labs (Imperial College, London).

During the Biomolecular aggregate session M. Di Carlo (CNR-Palermo) and M. Digman (CA Univ. at Irvine) have interested the participants in the effect on live cells of aggregation of beta-amyloid peptide and huntingtin, respectively.

The session of Processes and mechanisms of the biological membranes has had its main points in the lectures concerning the studies of membrane permeability of ion channels (A. Moroni, Milano Univ.) or to antimicrobial peptides (G. Rispoli, Ferrara Univ.).

D. Giacomazza $(\square)$

Institute of Biophysics,

National Research Council,

Via U. La Malfa 153, Palermo, Italy

e-mail: daniela.giacomazza@pa.ibf.cnr.it
For the session of Biomaterials and nano-biophysics O. Cavalleri (Genoa Univ.) and L. Cordone (Palermo Univ.) have discussed the possibility of a fine control of protein folding/unfolding to build molecular nanostructures or the preservation properties of trehalose-water matrixes, respectively.

The development of a framework for the registration of 3D imaging data for clinical heart investigations (R. Antolini, Trento Univ.) and the attempt to extend the results obtained on a charged, functionalized and patchy colloidal particles to globular protein solutions (F. Sciortino, Roma La Sapienza Univ.) have been discussed during the Theoretical and computational biophysics topic.

In the session Photobiophysics and photoprocesses, T. Kottke (Bielefeld Univ.) has discussed the mechanism of blue light reception in plant and animal cryptochromes and D. Dell'Orco (Modena and Reggio Emilia Univ.) the molecular systems biology approaches to phototransduction in vertebrate rods.

The investigation of a new biological scenario in which gene expression and regulation largely depend on a complex posttranscriptional network signals acting on mRNAs (A. Quattrone, Trento Univ.) and the relationship between structure and function in the P450 cytochrome involved in the erythromycin biosynthesis (B. Vallone, Roma La Sapienza Univ.) have been debated during the session regarding the Structure and function of proteins and nucleic acids.

The biophysical mechanisms of regulation of CLC chloride channels and transporters (M. Pusch, Genoa CNR) and the application of optical and fluorescence microscopies for dynamical tracking from cellular to molecular resolution (M. Faretta, Milan Eur. Inst. Oncol.) have been discussed during the Molecular and cellular biophysics session.

The last day of the meeting, four young scientists have received the prize for their research: 


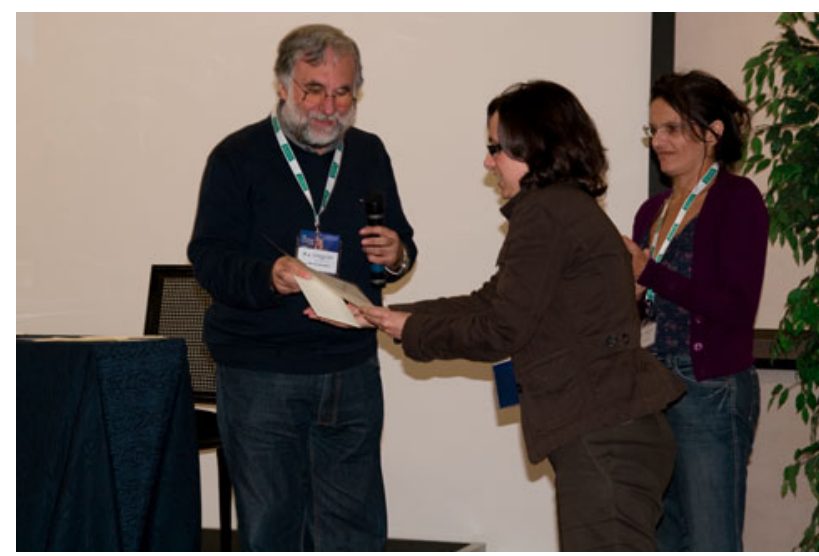

Fig. 1 Pier Luigi San Biagio (left), former president of the SIBPA and Silvia Morante (right), new president of the Society, during the awarding of prize of Lucia Di Giambattista Lattanzi

Susanna Alloisio (Genoa CNR) for her study on the native and recombinant $\mathrm{P} 2 \mathrm{X} 7$ as model for the receptor functional properties;
Christian Andersen (Copenhagen Novo Nordisk and Palermo CNR) for the investigation on the mechanism of fibrillation of hormone glucagon;

Lucia di Giambattista Lattanzi (Roma La Sapienza Univ.) for the survey of the effect induced by UV radiation on cells by FTIR spectroscopy (Fig. 1); and

Paolo Bianchini (Genoa Univ.) for the ex vivo analysis of retina obtained by confocal laser scanning imaging.

I would like to thank the scientific and organizing committees for their very valuable work and all the sponsors that have contributed to the success of the meeting.

I am also grateful to all authors and referees for their cooperation and support.

Special thanks goes to Anthony Watts and to the staff of EBJ for the expert suggestions in the fulfilment of the present special issue. 